\title{
ON AN APPLICATION OF INTERMEDIATE LOGICS
}

\author{
TOSHIO UMEZAWA
}

In [1] I investigated some logics intermediate between intuitionistic and classical predicate logics. The purpose of this paper is to show the possibility of applying some intermediate logics to mathematics namely, to show that some mathematical theorems which are provable in the classical logic but not provable in the intuitionistic logic are provable in some intermediate logics. Let $L Z$ be the logical system obtained from $L J^{\prime}$, a variant of Gentzen's $L J$ [2], by adding as axioms all those sequents which can be obtained from a sequent scheme $Z$ by substitution for propositional, predicate, or individual variables. Among many intermediate logics, $L M, L K^{\circ}, L M K^{\circ}, L E, L D$, and $L D^{*}$ are used in this paper where $M$ stands for $\rightarrow>\mathbf{A},>>\mathbf{A}, K^{\circ}$ for $\rightarrow>>\forall \mathbf{x}(\mathbf{A}(\mathbf{x}) \vee>\mathbf{A}(\mathbf{x})), M K^{\circ}$ for $\rightarrow>>\forall \mathbf{x A}(\mathbf{x}),>>\exists \mathbf{x}>\mathbf{A}(\mathbf{x}), E$ for $フ>\exists \mathbf{x A}(\mathbf{x}) \rightarrow \exists \mathbf{x}>>\mathbf{A}(\mathbf{x}), D$ for $\forall \mathbf{x}(\mathbf{A}(\mathbf{x})$ $\vee \mathbf{B}) \rightarrow \forall \mathbf{x A}(\mathbf{x}) \vee \mathbf{B}$, and $D^{*}$ for $\forall \mathbf{x}(\mathbf{A}(\mathbf{x}) \vee>\mathbf{B}) \rightarrow \forall \mathbf{x A}(\mathbf{x}) \vee>\mathbf{B}$. These are proper sublogics of the classical logic.

An interpretation of classical arithmetic is given in $\S 1$. Let us denote by $>>\Gamma$ the result of applying $>>$ to each formulas of $\Gamma$. Then it is proved that if $I \rightarrow \Delta$ is provable in $L K$, then $フ>I^{\prime} \rightarrow>>\Delta$ is provable in $L M K^{\circ}$.

In $\S 2$ we investigate an arithmetic of real number-generators. As for the intuitionistic terminologies not mentioned explicitly, we refer to Heyting [3]. Let us suppose that the intuitionistic theory of rational numbers has been developed. A sequence $\left\{a_{n}\right\}$ of rational numbers is called a $J$ - or $K^{\circ}$ - or $E$-(real) numbergenerator, if it satisfies $\forall k \exists n \forall p\left|a_{n+p}-a_{n}\right|<2^{-k},>>\forall k \exists n \forall p\left|a_{n+p}-a_{n}\right|$ $<2^{-k}$, or $\forall k>>\exists n \forall p\left|a_{n+p}-a_{n}\right|<2^{-k}$ where the ranges of variables are the set of all natural numbers. Any $J$-number-generator is a $K^{\circ}$-number-generator but not vice versa and any $K^{\circ}$-number-generator is $E$-number-generator but not vice versa. It is proved in Theorem 2 that in $L E$ as well as in $L D$ there is only one form of number-generator if we consider weakening in terms of double negation. Let $\alpha$ and $\beta$ be arbitrary number-generators. We say that $\alpha$ and $\beta$

Received October 21, 1959. 
$J$-coincide, if $\forall k \exists n \forall p\left|a_{n+p}-b_{n+p}\right|<2^{-k}$; that $\alpha$ is $J$-inequal to $\beta$, if $\exists k \forall n \exists p$ $\left|a_{n+p}-b_{n+p}\right| \geqq 2^{-k}$; and that $\alpha$ lies $J$-apart from $\beta$, if $\exists k \exists n \forall p\left|a_{n+p}-b_{n+p}\right|$ $\geqq 2^{-k}$. The other weakened forms of these notions in terms of double negation are given. In $L E$ as well as in $L D$ there is only one form of definition for coincidence, inequality, and apartness respectively and moreover inequality is an equivalent notion to apartness and negation of coincidence to apartness.

In $\S 3$ a definition of real numbers is given. Let $X, Y$, and $Z$ stand for one of $J, K^{\circ}$, and $E$. The species of $X$-real number-generators which $Y$-coincide with a given $Z$-real number-generator is called a $(X, Y, Z)$-real number. It is shown that there are nine forms of real numbers which are not known intuitionistically to be equivalent and relations of these forms of real numbers are given in a diagram. In $L E$ as well as in $L D$ there is only one form of real number.

In $\S 4$ irrational numbers are treated. A $J$-real number-generator $\left\{a_{n}\right\}$ is defined to be rational or irrational if $\exists r \forall k \exists n \forall p\left|a_{n+p}-r\right|<2^{-k}$ or $\forall r \exists k \forall n \exists p$ $\left|a_{n+p}-r\right| \geqq 2^{-k}$ where $r$ ranges over the set of rational numbers. It is proved in Theorem 6 that in $L D$ any real number is a $J$-rational number or a $J$-irrational number.

In $\S 5$ order relations are investigated. $\alpha<\beta$ means that $\exists k \exists n \forall p\left(a_{n+p}+2^{-k}\right.$ $<b_{n+p}$ ) where $a_{n+p}$ and $b_{n+p}$ are the $n+p$-th components of $\alpha$ and $\beta$ respectively. There are three weakened forms in terms of double negation which are not known intuitionistically to be equivalent and are equivalent in $L D$ as well as in $L E$. We obtain $\rightarrow \alpha>\beta \vee \alpha=\beta \vee \alpha<\beta$ in $L E$ as well as in $L D$. Let $\alpha \stackrel{*}{=} \beta$ be $E$-coincidence between $\alpha$ and $\beta$. In $L M$ as well as in $L D^{*} \rightarrow a \stackrel{*}{=} \beta \vee>(\alpha \stackrel{*}{=} \beta)$ is provable. Also it is proved that in $L M$ a virtual order relation is equivalent to an actual order relation.

Finally in $\S 6$ we investigate many forms of convergences of rational numbers and of real number-generators. Let $\left\{\alpha_{n}\right\}$ be a sequence of numbergenerators. $\left\{\alpha_{n}\right\}$ is called a $J$ - or $K^{\circ}$ - or $E$-fundamental sequence of the numbergenerators, if $\forall k \exists n \forall p\left|\alpha_{n+p}-\alpha_{n}\right|<2^{-k}, \quad>>\forall k \exists n \forall p\left|\alpha_{n+p}-\alpha_{n}\right|<2^{-k}$, or $\forall k>>\exists n \forall p\left|\alpha_{n+p}-\alpha_{n}\right|<2^{-k}$. The convergence of fundamental sequences of $J$-number-generators is treated. For any fundamental sequence $\left\{\alpha_{n}\right\}$ of any number-generators, it holds in $L E$ as well as in $L D$ that $\exists \alpha \forall k \exists n \forall p\left|\alpha-\alpha_{n+p}\right|$ $<2^{-k}$ where $\alpha$ ranges over $J$-number-generators. 


\section{$\S 1$. An interpretation of natural number theory}

Since the most part of classical elementary arithmetic is provable in the intuitionistic mathematics except some assertions like the principle of the least number, we here prove a theorem concerning an interpretation of classical arithmetic.

The system of intuitionistic elementary arithmetic is given by adding the following mathematical axiom sequents and the complete induction to $L J^{\prime}$. Terms and formulas are introduced as usual (cf. Kleene [4] Chap. IV).

$$
\begin{aligned}
& a=b \rightarrow a^{\prime}=b^{\prime} . \quad a^{\prime}=b^{\prime} \rightarrow a=b . \quad a^{\prime}=0 \rightarrow . \quad a=b, a=c \rightarrow b=c . \\
& \rightarrow a+0=a . \quad \rightarrow a+b^{\prime}=(a+b)^{\prime} . \quad \rightarrow a \cdot 0=0 . \quad \rightarrow a \cdot b^{\prime}=a \cdot b+a .
\end{aligned}
$$

Induction

$$
\underset{\Gamma \rightarrow A(0) \quad A(a), \Gamma \rightarrow A\left(a^{\prime}\right)}{\Gamma \rightarrow \forall x A(x)}
$$

where $a$ shall not occur in the lower sequent.

Let us denote the result of applying $>>$ to each formulas of $\Gamma$ by $>>I$ :

THEоRем 1. For the elementary arithmetic, if $\Gamma \rightarrow \Delta$ hola's classically, then $>>\Gamma \rightarrow>7 \Delta$ holds in $L M K^{\circ}$.

Proof. We use the induction on the length of a given classical proof. As for the sequent $A \rightarrow A$ it is obvious. It is also true as for the mathematical axiom sequents, because for natural numbers $a, b>7(a=b) \rightarrow a=b$ is true. We treat only $\forall$-in-succedent and Induction.

$\forall$-in-succedent. We omit evident inferences, inserting a double line to show a finite number of occurrences of inference.

$$
\begin{aligned}
& \text { フフ } \Gamma \rightarrow \text { フフ } \\
& \begin{array}{r}
>A(a),>>\Gamma \rightarrow 77 \Delta \\
\exists x>A(x),>7 \Gamma \rightarrow 77 \Delta
\end{array} \\
& 7 \Delta, \exists x>A(x), 7>\Gamma \rightarrow \\
& \text { フ }, \text { フフ } \Gamma \rightarrow \neg \exists x>A(x)
\end{aligned}
$$

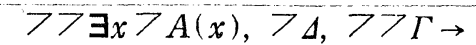

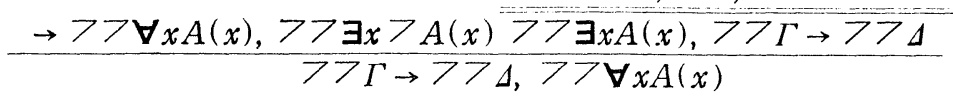

As for induction, we use the fact that $K^{\circ}$ is provable in $L M K^{\circ}$.

$$
\begin{aligned}
& \text { フフ } \Gamma \rightarrow \text { フ } A(0) \text { フフ } A(a), \text { フフ } \Gamma \rightarrow \text { フ } A\left(a^{\prime}\right) \\
& \text { フフ } \rightarrow \forall x \text { フ }
\end{aligned}
$$


Thus, the theorem has been proved.

\section{§ 2. Arithmetic of real number-generators}

The intuitionistic theory of rational numbers, including their order relation, is supposed to have been developed. In the following a mathematical sequent, which is intuitionistically provable, is admitted as an initial sequent.

Def. 1. A sequence $\left\{a_{n}\right\}$ of rational numbers is called a J-real numbergenerator, or shortly a $J$-number-generator, if for every natural number $k$ we can find effectively a natural number $n$, such that $\left|a_{n+p}-a_{n}\right|<2^{-k}$ for every natural number $p$ : written symbolically,

$$
\forall k \exists n \forall p\left|a_{n+p}-a_{n}\right|<2^{-k} .
$$

Unless mentioned otherwise, quantifiers range over the natural numbers.

We consider the forms of number-generators in which double negations stand before quantifiers. They are called weakened forms.

Def. 2. A sequence $\left\{a_{n}\right\}$ of rational numbers is called a $K^{\circ}$-(real) numbergenerator or $E$-(real) number-generator if it satisfies (2.2) or (2.3) respectively:

$$
\begin{aligned}
& \text { フ } \forall k \exists n \forall p\left|a_{n+p}-a_{n}\right|<2^{-n}, \\
& \forall k>>\exists n \forall p\left|a_{n+p}-a_{n}\right|<2^{-k},
\end{aligned}
$$

Any other weakened form is equivalent to one of these three. Obviously a $J$-number-generator is a $K^{\circ}$-number-generator, which is also a $E$-numbergenerator. We prove that (2.1) is a stronger condition than (2.2).

Let $M$ be a finitary spread consisting of all infinitely proceeding sequences in which only 0 and 1 can be members, while 1 can only be followed by 1 . By the definition of $M$ we can show that $\exists x\left(a_{x}=1\right) \rightarrow \forall k \exists n \forall p\left|a_{n+p}-a_{n}\right|<2^{-k}$ and $>\exists x\left(a_{x}=1\right) \rightarrow \forall k \exists n \forall p\left|a_{n+p}-a_{n}\right|<2^{-k}$, hence $\exists x\left(a_{x}=1\right) \vee>\exists x\left(a_{x}=1\right)$ $\rightarrow \forall k \exists n \forall p\left|a_{n+p}-a_{n}\right|<2^{-k}$. Since $\rightarrow>7\left(\exists x\left(a_{x}=1\right) \vee>\exists x\left(a_{x}=1\right)\right)$ is provable intuitionistically, we obtain $\rightarrow>>\forall k \exists n \forall p\left|a_{n+p}-a_{n}\right|<2^{-k}$. Hence, every element of $M$ is a $K^{\circ}$-number-generator. Assume that (2.2) entails (2.1). By the definition of $M(2.1)$ is equivalent to $\rightarrow \exists n \forall p\left|a_{n+p}-a_{n}\right|<2^{-1}$. If we take the least value of such $n$, then for any element $\alpha$ of $M$ a number is determined. We write the number as $f(\alpha)$. In virtue of the fan-theorem (cf. [3] p. 42), $f(\alpha)$ must be known after a finite number of components are given. However, it is impossible, q.e.d. 
An example which satisfies (2.3) but does not seem to satisfy (2.2) was given in [5].

Since $フ>\forall x A(x)$ is equivalent to $\forall x>>A(x)$ in $L K^{\circ}$, a $E$-number-generator is a $K^{\circ}$-number-generator. Therefore $K^{\circ}$-number-generator and $E$-numbergenerator are the same conception in $L K^{\circ}$. Kuroda [6] has developed a theory of real numbers on the assumption that $K^{\circ}$ is intuitionistically true if $x$ or every variable, which may eventually occur in the inner construction of $A(x)$, range over a denumerable infinite species, hence the real number theory in $L K^{\circ}$ (cf. [6], [7] and [8]).

THEOREM 2. In LE as well as in LD there is only one form of numbergenerator for weakening in terms of double negations.

Proof. If suffices to prove the sequent $\forall k>>\exists n \forall p\left|a_{n+p}-a_{n}\right|<2^{-k}$ $\rightarrow \forall k \exists n \forall p\left|a_{n+p}-a_{n}\right|<2^{-k}$ in $L E$ and in $L D$. Let us abbreviate $\left|a_{n+p}-a_{n}\right|$ $<2^{-k}$ by $\mathfrak{A}(k, n, p)$.

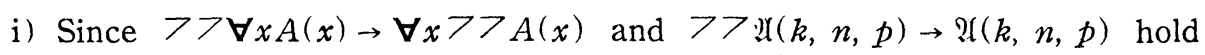
intuitionistically, we can obtain the desired result by applying $E$.

ii) The proof in $L D$ is as follows.

$\rightarrow \mathfrak{U}(k, n, p),>\mathfrak{U}(k, n, p)$

$\rightarrow \mathfrak{R}(k, n, p), \exists p>\mathfrak{R}(k, n, p) \quad \exists p>\mathfrak{A}(k, n, p) \rightarrow>\forall p \mathfrak{A}(k, n, p)$ $\begin{aligned} & \rightarrow \mathfrak{U}(k, n, p),>\forall p \mathfrak{A}(k, n, p) \\ \rightarrow \forall p \mathfrak{U}(k, n, p),>\nabla p \mathfrak{A}(k, n, p) & \end{aligned}$ $\rightarrow \exists n \forall p \mathfrak{N}(k, n, p),>\forall p \mathfrak{N}(k, n, p)$

$\rightarrow \exists n \forall p \mathfrak{A}(k, n, p), \forall n>\forall p \mathfrak{A}(k, n, p) \quad \forall n>\forall p \mathfrak{R}(k, n, p) \rightarrow>\exists n \forall p \mathfrak{Q}(k, n, p)$ $\rightarrow \exists n \forall p \mathfrak{Q}(k, n, p),>\exists n \forall p \mathfrak{R}(k, n, p)$

ᄀᄀ $\exists \forall \forall \mathfrak{A}(k, n, p) \rightarrow \exists n \forall p \mathfrak{R}(k, n, p)$

$\forall k>7 \exists n \forall p \mathfrak{A}(k, n, p) \rightarrow \exists n \forall p \mathfrak{N}(k, n, p)$
$\forall k>7 \exists n \forall p \mathfrak{I}(k, n, p) \rightarrow \forall k \exists n \forall p \mathfrak{A}(k, n, p)$

where $\rightarrow \mathfrak{Q}(k, n, p),>\mathfrak{A}(k, n, p)$ and other sequents are provable intuitionistically, q.e.d.

If in the following, a number-generator is denoted by a small Greek letter, then the number-generator shall be understood that it denotes any one of the above three forms. Let number-generators $\left\{a_{n}\right\}$ and $\left\{b_{n}\right\}$ be denoted by $\alpha$ and $\beta$ respectively.

Def. 3. $\alpha$ and $\beta$ J-coincide, $\alpha=\beta$, if

$$
\forall k \exists n \forall p\left|a_{\imath+p}-b_{n+p}\right|<2^{-k} .
$$


Def. 4. $\alpha$ and $\beta K^{\circ}$ - or E-coincide in case of (2.5) or (2.6) respectively.

$$
\begin{aligned}
& \text { フフ } \forall k \exists n \forall p\left|a_{n+p}-b_{n+p}\right|<2^{-k} . \\
& \forall k>>\exists n \forall p\left|a_{n+p}-b_{n+p}\right|<2^{-k} .
\end{aligned}
$$

We can prove in the same way as in Theorem 2 that in $L K^{\circ} K^{\circ}$-coincidence is equivalent to $E$-coincidence, and in $L E$ as well as in $L D, J$ - and $K^{\circ}$ - and $E$ coincidence are equivalent to each other.

Def. 5. $\alpha$ is $J$-inequal to $\beta$, if

$$
\exists k \forall n \exists p\left|a_{n+p}-b_{n+p}\right| \geqq 2^{-k} .
$$

There are other weakenings which are not known intuitionistically to be equivalent :

$$
\begin{aligned}
& \exists k>>\forall n \exists p\left|a_{n+p}-b_{n+p}\right| \geqq 2^{-k} . \\
& >>\exists k \forall n \exists p\left|a_{n+p}-b_{n+p}\right| \geqq 2^{-k} . \\
& \exists k \forall n>>\exists p\left|a_{n+p}-b_{n+p}\right| \geqq 2^{-k} . \\
& >>\exists k \forall n>>\exists p\left|a_{n+p}-b_{n+p}\right| \geqq 2^{-k} .
\end{aligned}
$$

In $L K^{\circ}$ there are three forms which are not known to be equivalent, i.e. (2.7), (2.8) and (2.9). In $L E$ as well as in $L D$ these five forms are equivalent to each other.

As for the relations among the different forms of coincidence, inequality and their negations in $L J^{\prime}$ or in $L K^{\circ}$, we refer to [5] or [6] respectively.

Def. 6. $\alpha$ lies J-apart from $\beta, \alpha \# \beta$, if

$$
\exists k \exists n \forall p\left|a_{n+p}-b_{n+p}\right| \geqq 2^{-k} .
$$

The other weakenings which are not known intuitionistically to be equivalent are as follows.

$$
\begin{aligned}
& \exists k>>\exists n \forall p\left|a_{n+p}-b_{n+p}\right| \geqq 2^{-k} . \\
& \exists n>>\exists k \forall p\left|a_{n+p}-b_{n+p}\right| \geqq 2^{-k} . \\
& >>\exists k \exists n \forall p\left|a_{n+p}-b_{n+p}\right| \geqq 2^{-k} .
\end{aligned}
$$

(2.14) is a weakening of an equivalent form $\exists n \exists k \forall p\left|a_{n+p}-b_{n+p}\right| \geqq 2^{-k}$ of (2.12).

We prove that for $J$-number-generators (2.12) is equivalent to (2.7) and for any number-generator (2.13) to $(2.8)$ and (2.15) to $(2.9)$, 
For any $\left\{a_{n}\right\}$, and $\left\{b_{n}\right\} \exists k \exists n \forall p\left|a_{n+p}-b_{n+p}\right| \geqq 2^{-k} \rightarrow \exists k \forall n \exists p\left|a_{n+p}-b_{n+p}\right|$ $\geqq 2^{-k}$ can be proved easily. The converse is proved for $J$-number-generators as follows. From the sequent $\left|a_{n+m+p}-a_{n}\right|<2^{-\imath-3},\left|a_{n+m+l}-a_{n}\right|<2^{-u-3}, \mid a_{n+m+l}$ $-b_{n+m+l}\left|<2^{-u-2},\right| b_{m+n+l}-b_{m}\left|<2^{-u-2},\right| b_{m+n+p}-b_{m}\left|<2^{-u-2} \rightarrow\right| a_{n+m+p}-b_{n+m+p} \mid$ $<2^{-u}$ we obtain $\forall p\left|a_{n+p}-a_{n}\right|<2^{-u-3}, \forall p\left|b_{m+p}-b_{m}\right|<2^{-u-2},\left|a_{n+m+p}-b_{n+m+p}\right|$ $\geqq 2^{-u} \rightarrow\left|a_{n+m+l}-b_{n+m+l}\right| \geqq 2^{-u-2}$ and further $\forall p\left|a_{n+p}-a_{n}\right|<2^{-u-3}, \forall p\left|b_{m+p}-b_{m}\right|$ $<2^{-u-2}, \quad \forall n \exists p\left|a_{n+p}-b_{n+p}\right| \geqq 2^{-u} \rightarrow \forall p\left|a_{n+m+p}-b_{n+m+p}\right| \geqq 2^{-u-2}$. Then $(\varepsilon)$ : $\exists n \forall p\left|a_{n+p}-a_{n}\right|<2^{-u-3}, \quad \exists n \forall p\left|b_{n+p}-b_{n}\right|<2^{-u-2}, \quad \forall n \exists p\left|a_{n+p}-b_{n+p}\right| \geqq 2^{-u}$ $\rightarrow \exists n \forall p\left|a_{n+p}-b_{n+p}\right| \geqq 2^{-\imath-2}$ is obtained. Hence, using $\rightarrow \forall k \exists n \forall p\left|a_{n+p}-a_{n}\right|$ $<2^{-k}$ and $\rightarrow \forall k \exists n \forall p\left|b_{n+p}-b_{n}\right|<2^{-k}$, we can obtain $\exists k \forall n \exists p\left|a_{n+p}-b_{n+p}\right|$ $\geqq 2^{-k} \rightarrow \exists k \exists n \forall p\left|a_{n+p}-b_{n+p}\right| \geqq 2^{-k}$. Therefore for $J$-number-generators (2.12) is equivalent to $(2.7)$.

$\exists k>>\exists n \forall p\left|a_{n+p}-b_{n+p}\right| \geqq 2^{-k} \rightarrow \exists k>>\forall n \exists p\left|a_{n+p}-b_{n+p}\right|<2^{-k}$ can be proved intuitionistically. From $(\varepsilon)$ we obtain $\forall k>>\exists n \forall p\left|a_{n+p}-a_{n}\right|<2^{-k}$, $\forall k>>\exists n \forall p\left|b_{n+p}-b_{n}\right|<2^{-k}, \quad \exists k>>\forall n \exists p\left|a_{n+p}-b_{n+p}\right| \geqq 2^{-k} \rightarrow \exists k>>\exists n \forall p$ $\left|a_{n+p}-b_{n+p}\right| \geqq 2^{-k}$. Then for $E$-number-generators, hence for any numbergenerators, $\exists k>>\forall n \exists p\left|a_{n+p}-b_{n+p}\right| \geqq 2^{-k} \rightarrow \exists k>>\exists n \forall p\left|a_{n+p}-b_{n+p}\right| \geqq 2^{-k}$ is provable. That is, (2.13) is equivalent to (2.8). The equivalence of $(2.15)$ to (2.9) can be proved similarly. $a \# b \rightarrow>(a=b)$ is provable intuitionistically but not conversely (cf. [3] p. 117). However, it can be proved that $>(a=b) \rightarrow a \# b$ holds in $L E$ as well as in $L D$. Hence we have

THEOREM 3. In LE as well as in LD there is only one form of definition for coincidence and inequality and apartness respectively. Inequality and apartness are equivalent notions and negation of coincidence is apartness.

\section{§3. Real numbers}

Let $X, Y$ and $Z$ stand for any one of $J, K^{\circ}$ and $E$.

Def. 7. The species of $X$-real number-generators which $Y$-coincide with a given $Z$-real number-generator is called a $(X, Y, Z)$-real number.

Lemma 1. Let $X$ stand for any one of $J, K^{\circ}$, and $E$. Any number-generator which $X$-coincide with a $X$-number-generator is a X-number-generator.

Proof. The sequent $\left|a_{m+n+l}-b_{m+n+l}\right|<2^{-u-2},\left|a_{n+m+l}-a_{n}\right|<2^{-u-2}, \mid a_{n+m}$ $-a_{n}\left|<2^{-u-2}, \quad\right| a_{m+n}-b_{m+n}\left|<2^{-u-2} \rightarrow\right| b_{n+m+l}-b_{n+m} \mid<2^{-u}$ is intuitionistically 
provable. From this we obtain $(\delta): \exists n \forall p\left|a_{n+p}-a_{n}\right|<2^{-\imath-2}, \exists n \forall p\left|a_{n+p}-b_{n+p}\right|$ $<2^{-u-2} \rightarrow \exists n \forall p\left|b_{n+p}-b_{n}\right|<2^{-u}$. Further by $\forall$-in-succedent and $\forall$-in-antecedent, we obtain ( $\varepsilon$ ): $\forall k \exists n \forall p\left|a_{n+p}-a_{n}\right|<2^{-k}, \forall k \exists n \forall p\left|a_{n+p}-b_{n+p}\right|<2^{-k} \rightarrow \forall k \exists n \forall p$ $\left|b_{n+p}-b_{n}\right|<2^{-k}$. Therefore if $\left\{a_{n}\right\}$ is a $J$-number-generator and $\left\{a_{n}\right\},\left\{b_{n}\right\} J$ coincide, then $\left\{b_{n}\right\}$ is a $J$-number-generator. From $(\varepsilon)$ we can obtain $フ>\forall k \exists n \forall p$ $\left|a_{n+p}-a_{n}\right|<2^{-k},>>\forall k \exists n \forall p\left|a_{n+p}-b_{n+p}\right|<2^{-k} \rightarrow>>\forall k \exists n \forall p\left|b_{n+p}-b_{n}\right|<2^{-k}$. Hence if $\left\{a_{n t}\right\}$ is a $K^{\circ}$-number-generator and $\left\{a_{n}\right\},\left\{b_{n}\right\} K^{\circ}$-coincide, then $\left\{b_{n}\right\}$ is a $K^{\circ}$-number-generator. Using $(\delta)$, we can prove that if $\left\{a_{n}\right\}$ is a $E$-numbergenerator and $\left\{a_{n}\right\},\left\{b_{n}\right\} E$-coincide, then $\left\{b_{n}\right\}$ is a $E$-number-generator, q.e.d.

Theorem 4. ( $\left.K^{\circ}, J, J\right)$-and $(E, J, J)$-real numbers and every $(J, X, Y)$-real number, in which $X$ and $Y$ stand for each of $J, K^{\circ}$ and $E$, are equal.

Proof. In virtue of Lemma 1 , all members of $\left(K^{\circ}, J, J\right)$ - and $(E, J, J)$-real number are $J$-number-generators, hence $\left(K^{\circ}, J, J\right)$ - and $(E, J, J)$-real numbers are equal to $(J, J, J)$-real numbers. Similarly $\left(J, J, K^{\circ}\right)$ - and $(J, J, E)$-real numbers are $(J, J, J)$-real numbers.

Since for $J$-number-generators $J-K^{\circ}$ - and $E$-coincidence are equivalent by [5] 2.51, $\left(J, K^{\circ}, J\right)$ - and $(J, E, J)$-real numbers are equal to $(J, J, J)$-real numbers.

$\left(J, K^{\circ}, K^{\circ}\right)-,\left(J, K^{\circ}, E\right)$ - and $\left(J, E, K^{\circ}\right)$-real numbers are $(J, E, E)$-real numbers. Let $\beta \equiv\left\{b_{n}\right\}$ and $\gamma \equiv\left\{c_{n}\right\}$ are arbitrary members of a $(J, E, E)$-real number. By definition, $\beta$ and $\gamma E$-coincide with a $E$-number-generator, say $\alpha \equiv\left\{\boldsymbol{a}_{n}\right\}$. We can prove easily that $\forall k>>\exists n \forall p\left|a_{n+p}-b_{n+p}\right|<2^{-k}, \forall k>>\exists n \forall p$ $\left|a_{n+p}-c_{n+p}\right|<2^{-k} \rightarrow \forall k>>\exists n \forall p\left|b_{n+p}-c_{n+p}\right|<2^{-k}$, therefore $\beta$ and $\gamma E$-coincide. Then by [5] $2.51 \beta$ and $\gamma J$-coincide, because $\beta$ and $\gamma$ are $J$-number-generators. Hence, if we consider a member, say $\delta \equiv\left\{d_{n}\right\}$, then all members of the $(J, E, E)$ real number $J$-coincide with $\delta$. Consequently any $(J, E, E)$-real number is a $(J, J, J)$-real number, q.e.d.

Def. 8. Real numbers in Theorem 4 are called J-real numbers.

Lemma 2. For $K^{\circ}$-number-generators $K^{\circ}$ - and E-coincidence are equivalent.

Proof. Let $\left\{a_{n}\right\}$ and $\left\{b_{n}\right\}$ be $K^{\circ}$-number-generators which $E$-coincide. From an intuitionistically provable sequent $\left|a_{n+m}-a_{n}\right|<2^{-u-4},\left|a_{n+m+l}-\dot{a}_{n}\right|<2^{-u-4}$, $\left|a_{n+m+l}-b_{n+m+l}\right|<2^{-u-2},\left|b_{m+n+l}-b_{m}\right|<2^{-u-4},\left|b_{m+n}-b_{m}\right|<2^{-u-4},\left|a_{n+m}-b_{n+m}\right|$ $\geqq 2^{-u-1} \rightarrow$ we can obtain $\forall k>>\exists n \forall p\left|a_{n+p}-b_{n+p}\right|<2^{-k}, \forall p\left|a_{n+p}-a_{n}\right|$ $<2^{-u-4}, \forall p ! b_{m+p}-b_{m}\left|<2^{-u-4},\right| a_{n+m}-b_{n+m} \mid \geqq 2^{-u-1} \rightarrow$. By the assumption, 
$\rightarrow \forall k>>\exists n \forall p\left|a_{n+p}-b_{n+p}\right|<2^{-k}$ is true. Using intuitionistically provable sequents $\rightarrow\left|a_{n+m}-b_{n+m}\right|<2^{-u-1},\left|a_{n+m}-b_{n+m}\right| \geqq 2^{-u-1}$ and $\left|a_{n+m+l}-a_{n}\right|<2^{-u-4}$, $\left|a_{n+m}-a_{n}\right|<2^{-u-4}, \quad\left|a_{n+m}-b_{n+m}\right|<2^{-u-1}, \quad\left|b_{m+n}-b_{m}\right|<2^{-u-4}, \quad\left|b_{m+n+l}-b_{m}\right|$ $<2^{-u-4} \rightarrow\left|a_{n+m+l}-b_{n+m+l}\right|<2^{-u}$, we obtain $\forall p\left|a_{n+p}-a_{n}\right|<2^{-u-4}, \forall p \mid b_{m+p}$ $-b_{m}\left|<2^{-u-4} \rightarrow\right| a_{n+m+l}-b_{n+m+l} \mid<2^{-\imath}$. From this, the sequent $>>\forall k \exists n \forall p$ $\left|a_{n+p}-a_{n}\right|<2^{-k}, \quad>>\forall k \exists n \forall p\left|b_{n+p}-b_{n}\right|<2^{-k} \rightarrow>>\forall k \exists n \forall p\left|a_{n+p}-b_{n+p}\right|$ $<2^{-k}$ can be obtained. Since, by the assumption, $\left\{a_{n}\right\}$ and $\left\{b_{n}\right\}$ are $K^{\circ}$-numbergenerators, we obtain $\rightarrow>>\forall k \exists n \forall p\left|a_{n+p}-b_{n+p}\right|<2^{-k}$, which means that $\left\{a_{n}\right\}$ and $\left\{b_{n}\right\} K^{\circ}$-coincide. $K^{\circ}$-coincidence entails $E$-coincidence, so the lemma has been proved.

Theorem 5. (I) $\left(K^{\circ}, J, K^{\circ}\right)$-, $\left(E, J, K^{\circ}\right)$-, and $\left(K^{\circ}, J, E\right)$-real numbers are equal. (II) $\left(K^{\circ}, K^{\circ}, J\right)-,\left(K^{\circ}, E, J\right)$, and $\left(E, K^{\circ}, J\right)$-real numbers are equal. (III) $\left(K^{\circ}, K^{\circ}, K^{\circ}\right)_{-,}\left(K^{\circ}, E, K^{\circ}\right)-,\left(E, K^{\circ}, K^{\circ}\right)_{-},\left(K^{\circ}, E, E\right)-$, and $\left(K^{\circ}, K^{\circ}, E\right)$ real numbers are equal.

Proof. $\left(K^{\circ}, J, K^{\circ}\right)$-real numbers are both $\left(E, J, K^{\circ}\right)$ - and $\left(K^{\circ}, J, E\right)$-real numbers. Since $J$-coincidence entails $K^{\circ}$-coincidence, $\left(E, J, K^{\circ}\right)$ - and $\left(K^{\circ}, J, E\right)$ real numbers are, in virtue of Lemma $1,\left(K^{\circ}, J, K^{\circ}\right)$-real numbers, hence (I) has been proved. It can be proved similarly, that $\left(K^{\circ}, K^{\circ}, J\right)$ - and $\left(E, K^{\circ}, J\right)$ real numbers are equal. By Lemma $2, E$-coincidence in $\left(K^{\circ}, E, J\right)$-real numbers is equivalent to $K^{\circ}$-coincidence, therefore (II) has been proved. In the same way (III) can be proved except for $\left(K^{\circ}, E, E\right)$-real numbers. The equivalence of $\left(K^{\circ}, E, E\right)$-real numbers to $\left(K^{\circ}, K^{\circ}, K^{\circ}\right)$-real numbers can be shown analogously as in $(J, E, E)$-real numbers, q.e.d.

Let us denote by $X \rightarrow Y$ that $X$-real numbers entail $Y$-real numbers. Then relations among real numbers of various forms are shown in the following diagram;

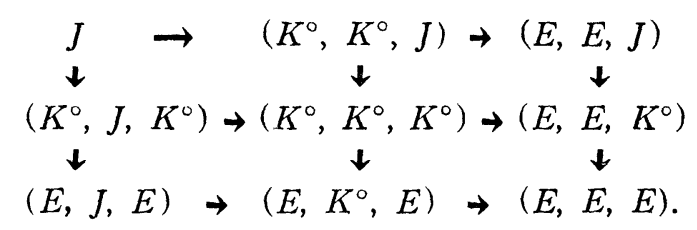

A $(E, E, E)$-real number was called a weak real number in [3] p. 111 .

It can be proved that in $L E$ as well as in $L D$ there is only one definition of real number. 


\section{§4. $J$-irrational number}

Def. 9. Let $\left\{a_{n}\right\}$ be a $J$-real number-generator. $\left\{a_{n}\right\}$ is called a J-rational number-generator, if

$$
\exists r \forall k \exists n \forall p\left|a_{n+p}-r\right|<2^{-k},
$$

in which $r$ is supposed to range over the rational numbers.

The species of $J$-number-generators which $J$-coincide with a $J$-rational number-generator is called a J-rational number.

Def. 10. Let $\left\{a_{n}\right\}$ be a $J$-real number-generator. $\left\{a_{n}\right\}$ is called a J-irrational number-generator, if

$$
\forall r \exists k \forall n \exists p\left|a_{n+p}-r\right| \geqq 2^{-k}
$$

where $r$ is supposed to range over the rational numbers.

The species of $J$-number-generators which $J$-coincide with a given $J$-irrational number-generator is called a $J$-irrational number.

Any sequence of rational numbers which $J$-coincides with a $J$-rational or $J$ irrational number generator is a $J$-rational or $J$-irrational number-generator respectively.

THEOREM 6. In LD any real number is a J-rational number or a J-irrational number.

Proof. Let $\alpha$ be an arbitrary member of a given real number. In virtue of Theorem 2, we may assume that $\alpha$ is a $J$-number-generator. In the same way as the foregoings, we can prove that in $L D \alpha$ is a $J$-rational numbergenerator or a $J$-irrational number-generator. If $\alpha$ is a $J$-rational numbergenerator, then the given real number is a $J$-rational number and its members are all $J$-rational number-generators. If $\alpha$ is a $J$-irrational number-generator, then the given real number is a $J$-irrational number.

\section{$\S 5$. Order relations}

Def. 11. For real number-generators $\alpha$ and $\beta, \alpha<\beta$, if

$$
\exists k \exists n \forall p\left(a_{n+p}+2^{-k}<b_{n+p}\right)
$$

where $a_{n+p}$ and $b_{n+p}$ are the $n+p$-th components of $\alpha$ and $\beta$ respectively. $\alpha>\beta$ means the same as $\beta<\alpha$. 
There are three weakenings which are not known intuitionistically to be equivalent, where (5.3) is a weakening of an equivalent form of (5.1).

$$
\begin{aligned}
& \exists k>>\exists n \forall p\left(a_{n+p}+2^{-k}<b_{n+p}\right) . \\
& \exists n>>\exists k \forall p\left(a_{n+p}+2^{-k}<b_{n+p}\right) . \\
& >>\exists k \exists n \forall p\left(a_{n+p}+2^{-k}<b_{n+p}\right) .
\end{aligned}
$$

Equivalences are also not known in $L K^{\circ}$. As in Theorem 2, we can prove that these four are equivalent to each other in $L E$ as well as in $L D$.

THEOREM 7. In LE as well as in LD there holds $\rightarrow \alpha=\beta \vee>(\alpha=\beta)$, hence by Theorem $3 \rightarrow \alpha=\beta \vee \alpha \approx \beta$.

The proof is similar to Theorem 2. Since $\alpha \neq \beta \rightarrow \alpha>\beta \vee \alpha<\beta$ is provable intuitionistically (cf. [3] p. 25), we obtain by Theorem 7

Corollary 7.1. In $L E$ as well as in $L D \rightarrow \alpha=\beta \vee \alpha>\beta \vee \alpha<\beta$. From this, we obtain further.

Corollary 7.2. In $L E$ as well as in $L D>(\alpha>\beta) \rightarrow \alpha=\beta \vee \alpha<\beta$ and $\alpha \neq \beta$ $\rightarrow>(\alpha>\beta) \vee>(\alpha<\beta)$ hold.

Proof. As to $7(\alpha>\beta) \rightarrow \alpha=\beta \vee \alpha<\beta$, it is obvious. From Corollary 7.1 $\alpha \neq \beta \rightarrow \alpha>\beta \vee \alpha<\beta$ follows. Since $\alpha>\beta \rightarrow>(\alpha<\beta)$ and $\alpha<\beta \rightarrow>(\alpha>\beta)$ are provable intuitionistically, the assertion can be obtained by cuts.

Def. 12. $\alpha \stackrel{\circ}{=} \beta$ denotes $K^{\circ}$-coincidence of $\alpha$ and $\beta$ and $\alpha \stackrel{*}{=} \beta E$-coincidence.

TheOREM 8. In $L M \rightarrow \alpha \stackrel{\ominus}{=} \vee>(\alpha \stackrel{\circ}{=} \beta)$ holds.

Proof. Obvious.

TheOREM 9. Im LM as well as in $L D^{*} \rightarrow \alpha \stackrel{*}{=} \beta \vee>(\alpha \stackrel{*}{=} \beta)$ holds.

Proof. Since $7>(\alpha \stackrel{*}{=} \beta)$ is equivalent to $\alpha \stackrel{*}{=} \beta$ intuitionistically, we obtain $\rightarrow \alpha \stackrel{*}{=} \beta \vee>(\alpha \stackrel{*}{=} \beta)$ from $\rightarrow>>(\alpha \stackrel{*}{=} \beta) \vee>(\alpha \stackrel{*}{=} \beta)$ in $L M$.

Let us abbreviate $\left|a_{n+p}-b_{n+p}\right|<2^{-k}$ by $\mathfrak{P}(k, n, p)$. The proof in $L D^{*}$ is as follows. From $\rightarrow \mathfrak{Y}(k, n, p),>\mathfrak{R}(k, n, p)$ we obtain $\rightarrow \mathfrak{P}(k, n, p)$, $>\forall p \mathfrak{Y}(k, n, p)$. Hence, by application of $D^{*}$, we obtain $\rightarrow \forall p \mathfrak{A}(k, n, p)$, $>\forall p \mathfrak{I}(k, n, p)$ and hence $\rightarrow>>\exists n \forall p \mathfrak{I}(k, n, p),>\forall p \mathfrak{A}(k, n, p)$. So, in terms of $D^{*}$, we obtain $\rightarrow>7 \exists n \forall p \mathfrak{U}(k, n, p), \forall n>\forall p \mathfrak{H}(k, n, p)$. Thence, $\rightarrow>>\exists n \forall p \mathfrak{A}(k, n, p),>\forall k>>\exists n \forall p \mathfrak{A}(k, n, p)$ holds. By application of $D^{*}$, we obtain $\rightarrow \forall k>>\exists n \forall p \mathfrak{U}(k, n, p),>\forall k>>\exists n \forall p \mathfrak{Y}(k, n, p)$. 
Def. 13. Let $S$ be a mathematical species. If $<$ is a predicate which satisfies the following rules, then $<$ is called a virtual order relation in $S$.

$$
\begin{aligned}
& a<b \rightarrow>(a>b) \wedge>(a=b) . \\
& a=b \wedge b<c \rightarrow a<c . \\
& a<b \wedge b=c \rightarrow a<c . \\
& a<b \wedge b<c \rightarrow a<c . \\
& >(a<b) \wedge>(a>b) \rightarrow a=b . \\
& >(a<b) \wedge>(a=b) \rightarrow a>b .
\end{aligned}
$$

$a>b$ means the same as $b<a$.

Def. 14. If $<$ satisfies the following rule instead of V5 and V6, then < is called an actual order relation in $S$.

$$
a=b \vee a>b \vee a<b .
$$

THEOREM 10. In LM a virtual order relation is the equivalent notion to an actual order relation.

Proof. An actual order relation is a virtual order relation. Conversely let us consider a virtual order relation. From V 1 and V6 we see that $a>b$ is equivalent to $>(a<b) \wedge>(a=b)$, therefore to $>(a<b \vee a=b)$. Hence $>>(a>b)$ is equivalent to フフフ $(a<b \vee a=b)$, consequently to $>(a<b \vee a$ $=b)$. Therefore $a>b$ is equivalent to $>>(a>b)$. From $\rightarrow>>(a>b),>(a>b)$ we can obtain $\rightarrow a>b,>(a>b)$ by a cut. Then by cuts of V 5 with $\rightarrow a<b$, $>(a<b)$ and $\rightarrow a>b,>(a>b)$, we can obtain $A$.

\section{$\S$ 6. Convergence}

Def. 15. Let $\alpha \equiv\left\{a_{n}\right\}$ and $\beta \equiv\left\{b_{n}\right\}$ be number-generators and let $c$ be a number. $\alpha-c, \alpha-\beta$ and $|\alpha|$ means $\left\{a_{n}-c\right\},\left\{a_{n}-b_{n}\right\}$ and $\left\{\left|a_{n}\right|\right\}$ respectively. $\alpha<c$ means $a<\left\{a_{n}\right\}$ where $a_{n}=c$.

Def. 16. A sequence $\left\{a_{n}\right\}$ of rational numbers is called $X 1-, X 2-, X 3-, X 4-$, or $X 5$-convergent, if $\left\{a_{n}\right\}$ satisfies the following condition respectively:-

$$
\begin{aligned}
& \exists \alpha \forall k \exists n \forall p\left|\alpha-a_{n+p}\right|<2^{-k}, \\
& \exists \alpha>>\forall k \exists n \forall p\left|\alpha-a_{n+p}\right|<2^{-k}, \\
& \exists \alpha \forall k>>\exists n \forall p\left|\alpha-a_{n+p}\right|<2^{-k},
\end{aligned}
$$




$$
\begin{aligned}
& >>\exists \alpha \forall k \exists n \forall p\left|\alpha-a_{n+p}\right|<2^{-k}, \\
& >>\exists \alpha \forall k>>\exists n \forall p\left|\alpha-a_{n+p}\right|<2^{-k}
\end{aligned}
$$

where $\alpha$ is supposed to range over the $X$-number-generators.

If $\left\{a_{n}\right\}$ is $X 1$-convergent, then it is $X 2$-convergent. If $\left\{a_{n}\right\}$ is $X 2$-convergent, then it is both $X 3$ - and $X 4$-convergent. If $\left\{a_{n}\right\}$ is either $X 3$ - or $X 4$-convergent, then it is $X 5$-convergent.

THEOREM 11. If $\left\{a_{n}\right\}$ is a J-, $K^{\circ}$, or E-number-generator, then $\left\{a_{n}\right\}$ is J1-, $K^{\circ} 2-$, or E3-convergent respectively and vice versa.

Proof. From the sequent $\left|a_{n+q}-a_{n}\right|<2^{-u-2},\left|a_{n+p}-a_{n}\right|<2^{-u-2} \rightarrow \mid a_{n+q}$ $-a_{n+p} \mid<2^{-u-1}$ we can obtain $\forall p\left|a_{n+p}-a_{n}\right|<2^{-u-2} \rightarrow \exists t \exists v \forall q\left(\left|a_{t+q}-a_{n+p}\right|\right.$ $\left.+2^{-v}<2^{-u}\right)$. It may be written as $\forall p\left|a_{n+p}-a_{n}\right|<2^{-u-2} \rightarrow\left|\alpha^{*}-a_{n+p}\right|<2^{-u}$, in which $\alpha^{*}$ is the sequence $\left\{a_{n}\right\}$. And further we obtain $(\delta): \forall k \exists n \forall p \mid a_{n+p}$ $-a_{n}\left|<2^{-k} \rightarrow \forall k \exists n \forall p\right| \alpha^{*}-a_{n+p} !<2^{-k}$. Hence, if $\alpha^{*} \equiv\left\{a_{n}\right\}$ is a $J$-numbergenerator, then $\rightarrow \forall k \exists n \forall p\left|\alpha^{*}-a_{n+p}\right|<2^{-k}$, consequently $\rightarrow \exists \alpha \forall k \exists n \forall p \mid \alpha$ $-a_{n+p} \mid<2^{-k}$. Namely, $\left\{a_{n}\right\}$ is $J 1$-convergent. From ( $\left.\delta\right)$ we can obtain $>>\forall k \exists n \forall p\left|a_{n+p}-a_{n}\right|<2^{-k} \rightarrow \exists \alpha>>\forall k \exists n \forall p\left|\alpha-a_{n+p}\right|<2^{-k}$. Hence, if $\alpha^{*}$ $\equiv\left\{a_{n}\right\}$ is a $K^{\circ}$-number-generator, then $\alpha^{*}$ is $K^{\circ} 2$-convergent. It can be proved in a similar way, that if $\alpha^{*} \equiv\left\{a_{n}\right\}$ is a $E$-number-generator, then $\alpha^{*}$ is E3convergent.

Conversely, from the sequent $\left|b_{t+s}-a_{n+m+l}\right|<2^{-u-1}-2^{-r},\left|b_{s+t}-a_{n+m}\right|$ $<2^{-u-1}-2^{-v} \rightarrow\left|a_{n+m+l}-a_{n+m}\right|<2^{-u}$, we can obtain $\exists t \exists r \forall q\left(\left|b_{t+q}-a_{n+m+l}\right|\right.$ $\left.+2^{-r}<2^{-u-1}\right), \quad \exists t \exists r \forall q\left(\left|b_{t+q}-a_{n+m}\right|+2^{-r}<2^{-u-1}\right) \rightarrow\left|a_{n+m+l}-a_{n+m}\right|<2^{-u}$. It may be written as $\left|\beta-a_{n+m+l}\right|<2^{-u-1},\left|\beta-a_{n+m}\right|<2^{-u-1} \rightarrow\left|a_{n+m+l}-a_{n+m}\right|<2^{-u}$. From this we can obtain $(\varepsilon): \forall k \exists n \forall p\left|\beta-a_{n+p}\right|<2^{-k} \rightarrow \forall k \mathbf{E} n \forall p\left|a_{n+p}-a_{n}\right|$ $<2^{-k}$. Since $\beta$ occurs free, the sequent $\exists \alpha \forall k \exists n \forall p\left|\alpha-a_{n+p}\right|<2^{-k} \rightarrow \forall k \exists n \forall p$ $\left|a_{n+p}-a_{n}\right|<2^{-k}$ follows. Hence, if $\left\{a_{n}\right\}$ satisfies (6.1), then $\left\{a_{n}\right\}$ is a $J$-numbergenerator. From ( $(\varepsilon)$, we can obtain $\exists \alpha>>\forall k \exists n \forall p\left|\alpha-a_{n+p}\right|<2^{-k} \rightarrow$ $>>\forall k \exists n \forall p\left|a_{n+p}-a_{n}\right|<2^{-k}$. Hence if $\left\{a_{n}\right\}$ satisfies (6.2), then $\left\{a_{n}\right\}$ is $K^{\circ}$. number-generator. It can be proved in a similar way, that if $\left\{a_{n}\right\}$ satisfies (6.3), then $\left\{a_{n}\right\}$ is a $E$-number-generator, q.e.d.

Remark. The statement of Theorem 11 still holds, if $<$ in the definitions (6.1) $-(6.5)$ is replaced by any one of the order relations defined by (5.2)-(5.4)

Def. 17. A sequence $\left\{\alpha_{n}\right\}$ of number-generators is called a $J_{-}, K^{\circ}-$, or $E$ - 
fundamental sequence, if $\left\{\alpha_{n}\right\}$ satisfies (6.6), (6.7), or (6.8) respectively:

$$
\begin{aligned}
& \forall k \exists n \forall p\left|\alpha_{n+p}-\alpha_{n}\right|<2^{-k}, \\
& >>\forall k \exists n \forall p\left|\alpha_{n+p}-\alpha_{n}\right|<2^{-k}, \\
& \forall k>>\exists n \forall p\left|\alpha_{n+p}-\alpha_{n}\right|<2^{-k} .
\end{aligned}
$$

Def. 18. A sequence $\left\{\alpha_{n}\right\}$ of $J$-number-generators is called $X 1-, X 2-, X 3-$, $X 4$-, or $X 5$-convergent, if $\left\{\alpha_{n}\right\}$ satisfies (6.9)-(6.13) respectively:

$$
\begin{aligned}
& \exists \alpha \forall k \exists n \forall p\left|\alpha-\alpha_{n+p}\right|<2^{-k}, \\
& \exists \alpha>>\forall k \exists n \forall p\left|\alpha-\alpha_{n+p}\right|<2^{-k} \text {, } \\
& \exists \alpha \forall k>>\exists n \forall p\left|\alpha-\alpha_{n+p}\right|<2^{-k} \text {, } \\
& >>\exists \alpha \forall k \exists n \forall p\left|\alpha-\alpha_{n+p}\right|<2^{-k} \text {, } \\
& \text { フフヨ } \alpha \forall k>>\exists n \forall p\left|\alpha-\alpha_{n+p}\right|<2^{-k},
\end{aligned}
$$

where $\alpha$ is supposed to range over the $X$-number-generators.

THEOREM 12. A $J-K^{\circ}$, and E-fundamental sequence of number-generators are $J 1-K^{\circ} 2$ - and E3-convergent respectively.

Proof. Let $\boldsymbol{a}_{n}^{(\boldsymbol{x})}$ be the $n$-th component of $\alpha_{x}$ in $\left\{\alpha_{n}\right\}$. We define $a^{(x)}$ to be $a_{n+1}^{(x)}$ where $n$ is the least number such that $\forall p\left|\alpha_{x}-a_{n+1}^{(x)}\right|<2^{-x}$. Then $\left|\alpha_{x}-a^{(x)}\right|$ $<2^{-x}$.

Let $d=\operatorname{Max}(m+1, u+2)$. The sequent $\exists n \forall p\left|\alpha_{d+l}-a_{n+p}^{(d+l)}\right|<2^{-(d+l)}, \exists n \forall p$ $\left|\alpha_{d}-a_{n+p}^{(d)}<2^{-d}, \forall p\right| \alpha_{m+p}-\alpha_{m}\left|<2^{-u-2} \rightarrow\right| a^{(d+l)}-a^{(d)} \mid<2^{-u}$ is provable. Since $>>\left|a^{(d+l)}-a^{(d)}\right|<2^{-u} \rightarrow\left|a^{(d+l)}-a^{(d)}\right|<2^{-u}$ holds intuitionistically, we can obtain $\forall k>>\exists n \forall p\left|\alpha_{k}-a_{n+p}^{(k)}\right|<2^{-k}, \forall p\left|\alpha_{m+p}-\alpha_{m}\right|<2^{-u-2} \rightarrow\left|a^{(d+l)}-a^{(d)}\right|<2^{-u}$. Since $\alpha_{k} \equiv\left\{a_{n}^{(k)}\right\}$ is a number-generator, $\rightarrow \forall k>>\exists n \forall p\left|\alpha_{k}-a_{n+p}^{(k)}\right|<2^{-k}$ is provable, as is seen from the proof of Theorem 11. Therefore we have $\forall p\left|\alpha_{m+p}-\alpha_{m}\right|$ $<2^{-u-2} \rightarrow\left|a^{(d+l)}-a^{(d)}\right|<2^{-u}$, hence $\exists n \forall p\left|\alpha_{n+p}-\alpha_{n}\right|<2^{-u-2} \rightarrow \exists n \forall p \mid a^{(n+p)}$ $-a^{(n)} \mid<2^{-u}$. From this, we can prove that $(A):$ if $\left\{\alpha_{n}\right\}$ is a $X$-fundamental sequence, then $\left\{a^{(n)}\right\}$ is a $X$-number-generator where $X$ stands for each of $J, K^{\circ}$, and $E$.

Let $g=\operatorname{Max}(n, u)$. The sequent $\exists n \forall p\left|\alpha_{g+l}-a_{n+p}^{(g+l)}\right| 2^{-(g+l)},\left|\alpha-a^{(g+l)}\right|$ $<2^{-u-1} \rightarrow\left|\alpha-\alpha_{g+l}\right|<2^{-u}$ is provable. As before we can obtain $\forall k>>\exists n \forall p$ $\left|\alpha_{k}-a_{n+h}^{(k)}\right|<2^{-k},\left|\alpha-a^{(g+l)}\right|<2^{-\imath-1} \rightarrow\left|\alpha-\alpha_{g+l}\right|<2^{-\imath}$. Since $\rightarrow \forall k>>\exists n \forall p$ $\left|\alpha_{k}-a_{p+n}^{(k)}\right|<2^{-k}$ holds, we obtain $\left|\alpha-a^{(g+l)}\right|<2^{-u-1} \rightarrow\left|\alpha-\alpha_{g+l}\right|<2^{-u}$. Using $g \geqq n$, we can obtain $\exists n \forall p\left|\alpha-a^{(n+p)}\right|<2^{-u-1} \rightarrow \exists n \forall p\left|\alpha-\alpha_{n+p}\right|<2^{-u}$. From 
this, it can be proved that if $\left\{a^{(n)}\right\}$ is $J 1-, K^{\circ} 2 \cdot$, or $E 3$-convergent, then $\left\{\alpha_{n}\right\}$ is $J 1-, K^{\circ} 2$, or $E 3$-convergent. In virtue of Theorem 11 and $(\mathrm{A})$, the theorem follows, q.e.d.

Since $>>|\alpha-a|<2^{-k} \rightarrow|\alpha-a|<2^{-k}$ and $7>|\alpha-\beta|<2^{-k} \rightarrow|\alpha-\beta|<2^{-k}$ are provable in $L E$ as well as in $L D$, it can be proved that $(6.1)-(6.5)$, that (6.6)-(6.8), and that $(0.9)-(6.13)$, are equivalent to each other.

THEOREM 13. In LE as well as in LD any fundamental seguence of numbergenerators is J1-convergent.

\section{REFERENCES}

[1] T. Umezawa, On logics intermediate between intuitionistic and classical predicate logics. J. Symbolic Logic 24 (1959), forthcoming.

[2] G. Gentzen, Untersuchungen über das logische Schließen. Math. Zeit. 39 (1934-5), $176-210$ and $405-431$.

[3] A. Heyting, Intuitionism. Amsterdam, 1956.

[4] S. C. Kleene, Introduction to metamathematics. Amsterdam-Groningen-New York 1952.

[5] M. Ohnishi, On intuitionistic functional calculus. Osaka Math. J. 5 (1953), 203-209.

[6] S. Kuroda, A study on the foundation of analysis (Japanese). Kiso-Kagaku 12 (1949), 1-12.

[7] S. Kuroda, Intuitionistische Untersuchungen der formalistischen Logik. Nagoya Math. J. 2 (1951), 35-47.

[8] S. Kuroda, On the intuitionistic and formalistic theory of real numbers. Proc. intern. Congress Math. 1954, Amsterdam. vol. 1.

\section{Mathematical Institute}

Nagoya University 